\title{
The Power of Correlative Microscopy - Understanding Deformation Compatibility with HR-EBSD and HR-DIC
}

\author{
Jun Jiang $^{1}$, Tiantian Zhang ${ }^{1,2}$, Fionn Dunne ${ }^{1}$ and Ben Britton ${ }^{1}$ \\ 1. Department of Materials, Imperial College London, London, SW7 2AZ \\ 2. current address: Department of Mechanical Engineering, Worcester Polytechnic Institute
}

New microscopy techniques afford us even more opportunities to understand the behaviour of real materials and ultimately link properties, microstructure and performance. The emergence of high angular resolution electron backscatter diffraction (HR-EBSD) [1] enables probing of heterogeneous elastic strain and lattice rotation with high precision. In a similar vein, high spatial resolution digital image correlation (HR-DIC) tracks changes between two or more images, through the application of a surface speckle pattern. The resultant displacement field can be interrogated to reveal high spatial resolution field maps of the total strain and rotation between images (with limited precision).

In this study, we use both techniques and focus on the incremental deformation of a single crystal nickel based superalloy during monotonic deformation. HR-DIC captures surface based components of the deformation tensor, and these fields reveal that strain localises to individual slip bands, evidenced in fields of total strain and in plane rotation (Figure 1 - top), which progressively develop as the sample is strained. The total deformation field results are used to verify whether compatibility is satisfied and that there is closure of the deformation field. HR-EBSD captures elastic strain gradients and elastic lattice rotation gradients, revealing heterogeneity associated with the presence of individual slip bands. Furthermore, as this sample deforms in single slip, GND density for the individual slip system is overdetermined and can be calculated from three independent measures of the elastic strain gradient fields measured with HR-EBSD. Perhaps surprisingly, we find that there is no appreciable increase in GND density within this sample (Figure 1 - bottom).

We rationalise this observation, the presence of total strain gradients from HR-DIC measurements and the lack of GND density measured with HR-EBSD, with the precise nature of unconstrained single slip. Fundamentally dislocation glide on a single slip system may not lead to an appreciable increase in GND density, but may result in appreciable total strain gradients (for some geometries). Fundamentally, our study verifies that compatibility is conserved in single slip and that slip may not result in the accumulation of GND density. This is important when considering the scope of EBSD measurements to evaluate strain in real materials. [3]

\section{References:}

[1] Wilkinson et al. Journal of Strain analysis (2010)

[2] Jiang, J. et al. Proceedings of the Royal Society A (2016)

[3] The authors are very grateful for the financial support provided by AVIC-BIAM, Rolls-Royce, EPSRC (grant EP/L025213/1), Royal Academy of Engineering for Dr Britton's RAEng of Research Fellowship and Prof Dunne's RAEng of a Research Chair. The authors gratefully acknowledge provision of the single crystal Ni superalloy sample by Prof Barbra Shollock 

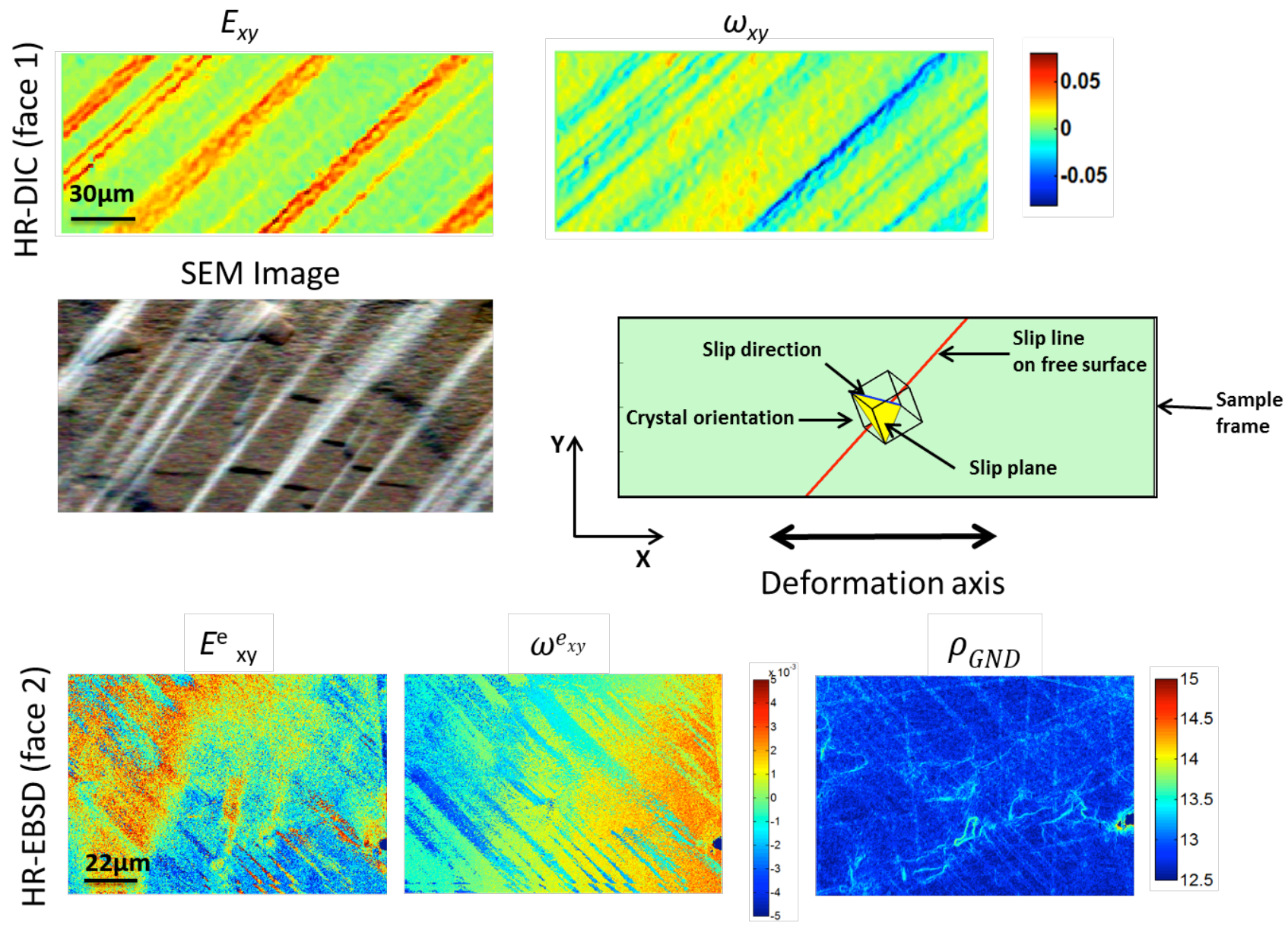

Figure 1. Deformation fields from deformed Ni sample aligned for single slip: (top) example HR-DIC showing strain localization within one slip planes and large total strain and rotation values; (bottom) example HR-EBSD showing elastic shear strain, lattice rotation and GND fields. 